\title{
Prophylaxe oder Prävention?
}

\author{
Diese beiden Begriffe bedeuten im Grunde dasselbe, nämlich Vorbeugung. "Prophylaxe“ ist aus \\ dem Griechischen und "Prävention" aus dem Lateinischen entlehnt. Doch die gesetzlichen und \\ privaten Krankenkassen honorieren in dem Bereich unterschiedliche Leistungen.
}

Unter Prophylaxe werden im Praxisalltag die zahnmedizinischen Bemühungen um effektive Mundhygiene sowie Zahnund Mundgesundheit verstanden. In der GKV ist das Spektrum der angebotenen Leistungen mit Befundung (IP1), Motivation (IP2), Fluoridapplikation (IP4) und Fissurenversiegelung (IP5) überschaubar, jedoch im Privatbereich auch nicht wesentlich umfangreicher. Dort sind in der Gebührenordnung für Zahnärzte (GOZ) zusätzlich die Leistungen Fluoridierungsschiene (1030), professionelle Zahnreinigung (1040) und Glattflächenversiegelung (2000 GOZ) zu finden.

Wenn man auf das Spektrum der Prophylaxeleistungen schaut, die BEMA und GOZ anbieten, dann handelt es sich dabei mit einer Ausnahme um Kariesprophylaxe. Die Ausnahme ist die „professionelle Zahnreinigung“ (PZR), die trotz Ansiedlung im GOZ-Abschnitt „Prophylaktische Leistungen“ in erster Linie eine parodontalprophylaktische Leistung darstellt. Das ist aber nur der eine Aspekt der PZR, der andere ist eine Gingivitis- und initiale Parodontitistherapie.

Die Vorbeugung vor Parodontalerkrankungen ist bei Gebrauch des Begriffs Prophylaxe nicht so präsent. Das ist nicht überraschend, fehlt doch zumindest in der GKV ein entsprechendes parodontal-prophylaktisches Angebot des BEMA.

Die Begriffsbildung „Parodontalprävention“ ist im Privatbereich selten, jedoch im GKV-Bereich völlig ungebräuchlich. Prävention in der Zahnmedizin wird eher so gesehen, dass Prävention ein Gesichtspunkt ist, der alle Bereiche der Zahnmedizin

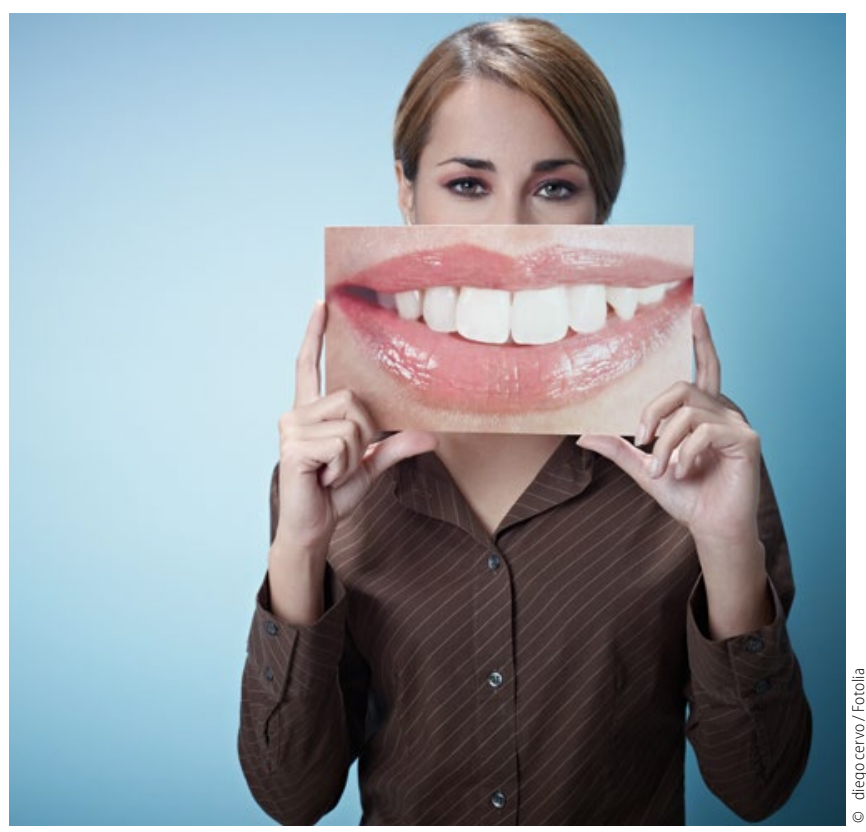

betrifft. Konservierende Zahnheilkunde zum Beispiel muss zugleich Prävention sein, soll etwa Zahnverlust vermeiden. Wie die an sich gleichbedeutenden Begriffe Prophylaxe und Prävention benutzt werden, zeigt Defizite in beiden Gebührenordnungen auf. Die Idee, konsequent die vorbeugenden GKV-Leistungen unter den Begriff Prophylaxe einzuordnen und die privaten Leistungen unter Prävention, ist organisatorisch gegebenenfalls hilfreich, für die Indikationsstellung aber ohne Belang.

\section{„Präventionsbehandlung"}

Von Belang ist jedoch das Problem, dass im Privatbereich nicht wenige private Krankenversicherungen ausdrücklich prophylaktische Leistungen gänzlich von der Erstattung ausschließen. Das Problem besteht seit Jahrzehnten, wird aber von Jahr zu Jahr etwas geringer. Die kurzsichtige Grundeinstellung mancher Krankenversicherungen ist, dass sie keine „Vorbeugungsversicherungen" sein wollen und auch gemäß den bundesrechtlichen Musterbedingungen für private Krankenkassen nicht dazu gezwungen sind. In diesen Fällen ist die Abgrenzung klar: Präventionsbehandlung ist kurative Frühbehandlung erster manifester Schäden, gegebenenfalls nur minimaler Veränderungen. Sind diese aber noch nicht feststellbar, besteht keine Erstattungspflicht.

Unter Präventionsbehandlung einzuordnen ist dann neben der Flächenversiegelung nach Nr. $2000 \mathrm{GOZ}$ von veränderten vestibulären - selten oralen - Zahnflächen auch eine sogenannte Icon-Behandlung (Kompositinfiltration) initialer Karies. In der Kieferorthopädie gibt es diesbezüglich eine nicht unerhebliche Anzahl von Diagnosen, deren Behandlung gemäß Richtlinien und KIG-Einstufung keine GKV-Leistungen darstellen, jedoch im privaten Behandlungsvertrag nicht abgetan werden können als zu frühe, rein „prophylaktische“ (überbesorgte) und zahnmedizinisch nicht notwendige Intervention. Therapie mit Aufbissbehelfen ist ein weiteres derartiges Beispiel.

Die ZA-Zahnärztliche Abrechnungsgenossenschaft eG hat zwei Folder zum QM bei Prophylaxe- und PAR-Abrechnung gedruckt, die in begrenzter Anzahl erhältlich sind.

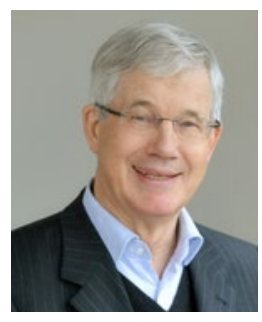

Dr. Peter H.G. Esser

GOZ-Experte und -Berater der ZA eG www.za-abrechnung.de 